\title{
Audit of Antibiotic Prescribing Practices for Neonatal Sepsis and Measurement of Outcome in New Born Unit at Kenyatta National Hospital
}

\author{
Priti Jagdishbhai Tank $(\mathbb{D}$, Anjumanara Omar, and Rachel Musoke \\ Department of Paediatrics and Child Health, School of Medicine, University of Nairobi, Kenya \\ Correspondence should be addressed to Priti Jagdishbhai Tank; priti.tank1984@gmail.com
}

Received 31 October 2018; Accepted 1 April 2019; Published 28 April 2019

Academic Editor: Alessandro Mussa

Copyright (C) 2019 Priti Jagdishbhai Tank et al. This is an open access article distributed under the Creative Commons Attribution License, which permits unrestricted use, distribution, and reproduction in any medium, provided the original work is properly cited.

\begin{abstract}
Background. Neonatal sepsis is a leading cause of morbidity and mortality globally. A high index of suspicion is required since features of sepsis are nonspecific. Auditing of antibiotic use is necessary to reduce misuse and minimise development of antibiotic resistance. Objectives. To assess the antibiotic prescribing practices in NBU at KNH against recommended Kenyan guidelines for neonatal sepsis. In addition, outcome within 7 days was described. Methods. This was a prospective audit of 320 neonates over a 2-month period at NBU of KNH. Data were collected using a structured questionnaire, stored in MS-EXCEL, and analysed using STATA. Results. Documentation of perinatal risk factors and clinical features at admission and at the time of change of antibiotics was very poor. The rate of investigations to confirm infection was very low. Blood cultures were done only in 13 (4\%) neonates on admission, while complete blood count and C reactive protein were done in 224 (70\%) and 198 (62\%), respectively. Appropriate antibiotics as per the Kenyan guidelines were prescribed in $313(97.8 \%)$ of neonates on admission. However, these were not stopped at 48-72 hours for the 148 (53.62\%) who had improved. Overall mortality was high in neonates at 80 (25\%). Majority (55\%) died within 48 hours. Mortality was high among preterm neonates; 70 (43.8\%) died out of 160. Conclusion. Overall documentation and investigations to confirm infection was poor. The continuation of antibiotics was inappropriate. Overall mortality was high especially in the first 48 hours of admission. To improve documentation, availability of a checklist on admission is recommended.
\end{abstract}

\section{Introduction}

According to Global Health Observatory data, 2.6 million neonates died in 2016. The main causes of neonatal deaths are prematurity and low birth weight, infections, asphyxia, and birth trauma. Sepsis accounts for around one-third deaths in neonates worldwide [1]. Survivors of neonatal sepsis are at higher risk of neurodevelopmental impairment [2-5].

Neonatal sepsis usually has nonspecific presentation, hence, delay in treatment, and especially initiation of effective antibiotic therapy results in serious consequences ranging from neurodevelopmental deficits to death [6]. Therefore, clinicians are urged to start empiric antibiotics to symptomatic neonates or neonates at high risk of sepsis while awaiting culture results [7]. Judicious use of antibiotics can be life-saving; however, both broad-spectrum antibiotics and prolonged treatment with empiric antibiotics can lead to development of antimicrobial resistance $[8,9]$. Prolonged duration of antibiotics can be associated with adverse outcomes like necrotising enterocolitis, late-onset sepsis, and death [10]. Antibiotic prescribing practices should be evaluated periodically for its rational use to prevent emergence of resistance [8].

In an effort to reduce neonatal mortality rate, Ministry of Health of Kenya published Basic Paediatric Protocols (February-2016), which has guidelines for management of neonatal sepsis [11]. Training of health personnel on use of the Kenyan guidelines is done through the emergency triage and treatment plus (ETAT+) course. Adherence to guidelines in terms of choice and duration of antibiotics is very necessary in order to reduce development and spread of resistance in the hospital, as well as in the community $[8,12]$. Antibiotics are 


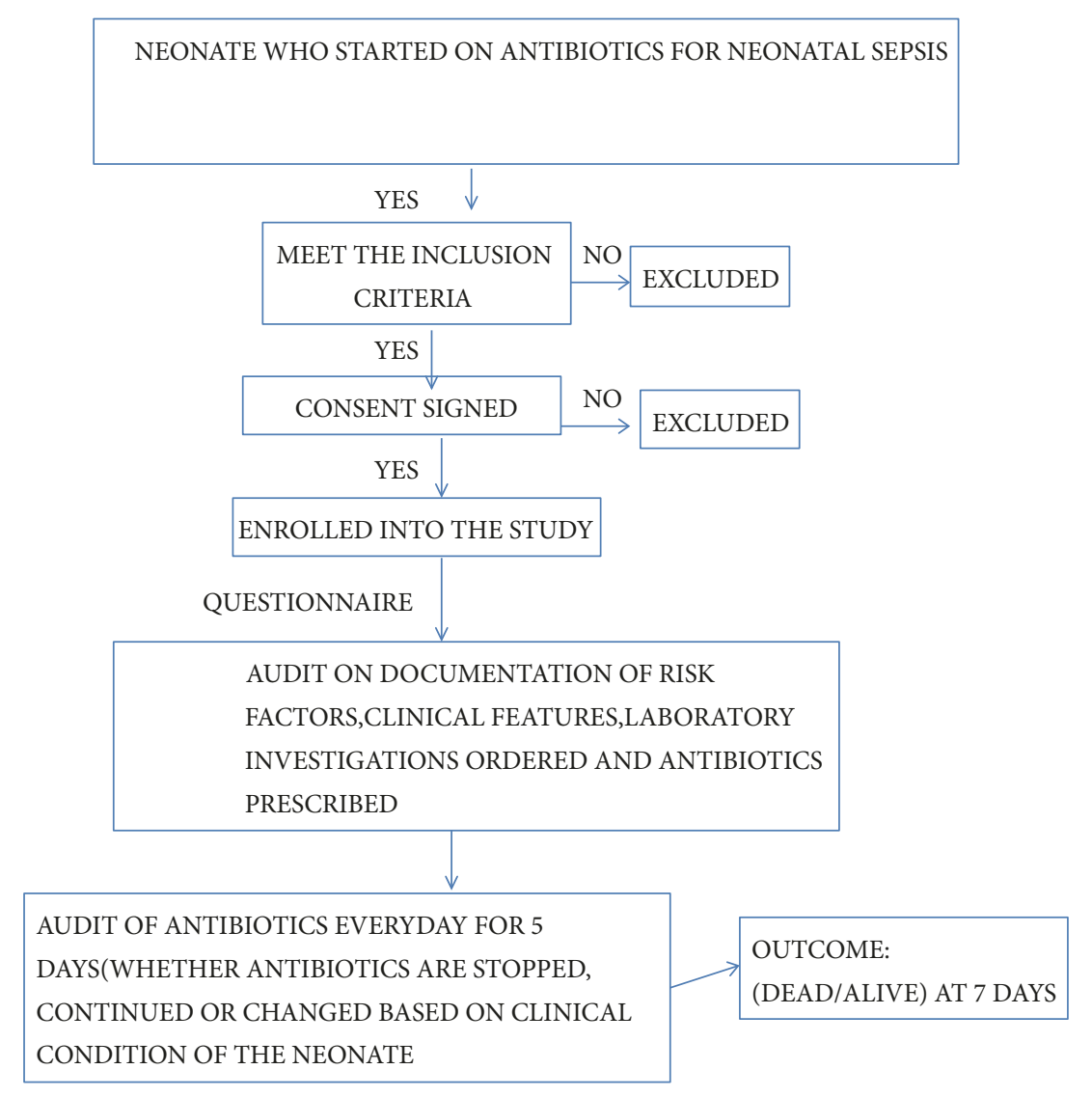

FIGURE 1: Study flowchart.

the most frequently prescribed medicines in newborn unit (NBU) at Kenyatta National Hospital (KNH) but audit of use has not been done. The primary objective of the current study was to assess the antibiotic prescribing practices in NBU at $\mathrm{KNH}$ against recommended Kenyan guidelines for neonatal sepsis.

\section{Methodology}

This was a prospective audit carried out over a period of 2 months in the Newborn Unit of Kenyatta National Hospital. $\mathrm{KNH}$ is a national tertiary referral and teaching hospital and the NBU has some facility for critical care. As a general rule all neonates with birth weight below $2000 \mathrm{~g}$ are admitted while those above that weight are only admitted if they require special care. About $30 \%$ of all admissions are referrals from other health facilities. The average admission rate is about 250 neonates per month.

The sample size was determined using Fischer's Formula and a sample size of 320 was taken. Population correction factor was applied for finite population. Data were collected daily from the neonate's medical record by PJT with assistance of two clinical officers using a standardised questionnaire. Consecutive sampling of neonates who were started on any antibiotics within the NBU was done. Neonates with birth asphyxia, major congenital malformations, and referred neonates who had received antibiotics for $>48$ hours were excluded. Collected data were recorded in the computer storage program MS-EXCEL at the end of 7th day of followup of the enrolled participant. Data verification was done manually for each record by PJT. To prevent inappropriate use access to the data required a password. Figure 1 shows the flow of data collection.

2.1. Statistics. Data analysis was done by STATA software package. Continuous variables were summarised using means (Standard Deviation) and medians (range). Categorical variables were presented as frequency distributions using tables or graphs. We computed the proportion of neonates whose prescription conforms to the national recommendations. The data collected on the audit of antibiotic prescribing practices were compared with the recommended Kenyan guidelines. The chi square test was used to compare the outcome variable (dead or alive at 7 days) versus categorical variables across two groups by level of guideline adherence. All independent variables were combined to generate regression models to compare effect on the outcome variable. The proportion of correct antibiotic prescribing practices determined 95\% confidence interval. The proportion of neonates dead or alive at 7 days or on discharge determined $95 \%$ confidence interval. 
TABLE 1: Socio-demographic characteristics of participants.

\begin{tabular}{|c|c|c|}
\hline Variable & Characteristics & Frequency (\%) $n=320$ \\
\hline \multirow{5}{*}{ Gestational age(in weeks) } & $<28$ & $17(5.3)$ \\
\hline & $28-<32$ & $31(9.7)$ \\
\hline & $32-<37$ & $112(35)$ \\
\hline & $37-40$ & $157(49.1)$ \\
\hline & $>42$ & $3(0.9)$ \\
\hline \multirow{2}{*}{ Age at admission(in days) } & $1-3$ & $297(92.8)$ \\
\hline & $\geq 4$ & $23(7.2)$ \\
\hline \multirow{2}{*}{ Sex } & Male & $170(53.2)$ \\
\hline & Female & $150(46.8)$ \\
\hline \multirow{5}{*}{ Birth weight(in grams) } & $<1000$ & $16(5)$ \\
\hline & $1000-<1500$ & $36(11.3)$ \\
\hline & $1500-<2500$ & $110(34.4)$ \\
\hline & $2500-<4000$ & $150(46.8)$ \\
\hline & $\geq 4000$ & $8(2.5)$ \\
\hline \multirow{2}{*}{ Referral from another facility } & Yes & $134(42)$ \\
\hline & No & $186(58)$ \\
\hline \multirow{2}{*}{ Place of delivery } & Hospital & $307(96)$ \\
\hline & Home & $13(4)$ \\
\hline \multirow{3}{*}{ Mode of delivery } & SVD & $147(46)$ \\
\hline & $\mathrm{C} / \mathrm{S}$ & $160(50)$ \\
\hline & Breech & $13(4)$ \\
\hline
\end{tabular}

TABLE 2: Audit of documentation of clinical features.

\begin{tabular}{lc}
\hline Clinical features(not documented) & Frequency \% $(\mathrm{n}=320)$ \\
\hline Convulsions & $252(41.1)$ \\
Lethargy & $65(10.6)$ \\
Bulging fontanel & $35(5.7)$ \\
Lower chest wall indrawing & $76(12.4)$ \\
Grunting & $128(20.9)$ \\
Cyanosis & $24(3.9)$ \\
Pallor & $18(2.9)$ \\
Jaundice & $16(2.6)$ \\
\hline
\end{tabular}

2.2. Ethics. A written informed consent was obtained from the parent/guardian. Approval was obtained from the KNH/UON ethics committee.

\section{Results}

3.1. Sociodemographic Characteristic of Study Population. A total of 320 neonates aged 0 day to 28 days admitted were enrolled in the study. The median age at admission was 1 day (IQR 1-7 days) and 297 (92.8\%) were aged between 1 and 3 days. Neonates who received antibiotics for presumed early-onset sepsis were 297 (92.8\%), while 23 (7.2\%) for lateonset sepsis. Table 1 shows sociodemographic characteristics of 320 enrolled neonates. It was noted that there was equal distribution between preterm and term neonates.
3.2. Audit of Documentation of Clinical Features. Complete documentation of clinical features for neonatal sepsis was not done in any of the 320 neonates. The clinical features not documented by clinician on admission were convulsions in 252 (41.05\%), grunting in 128 (20.85\%), lower chest wall indrawing in 76 (12.4\%), and lethargy in 65 (10.6\%) as shown in Table 2. Signs of respiratory distress were mostly present and documented, because study population included preterm neonates as well. Refusal to breastfeed was documented in a few term neonates admitted from postnatal wards of $\mathrm{KNH}$.

3.3. Audit of Documentation of Perinatal Risk Factors. Overall documentation of perinatal risk factors was very poor. As mentioned in Table 3, the most commonly documented perinatal risk factors were low birth weight (documented in $100 \%$ ), prolonged rupture of membranes (documented in $51.6 \%$ ), suspected or confirmed chorioamnionitis (documented in $21.8 \%$ ) and difficult or prolonged labour (documented in $7.8 \%$ ). Maternal fever was documented only in $1.6 \%$. Only $2(0.6 \%)$ had history of maternal intrapartum antibiotics use. Overall 53(16.6\%) neonates had maternal risk factors present.

3.4. Audit of Investigations Done on Admission. Blood cultures were done only in 13(4\%) neonates on admission, while complete blood count and $\mathrm{C}$ reactive protein were done in $224(70 \%)$ and $198(62 \%)$ respectively as shown in Table 4. Blood sugar was measured in $26(8 \%)$ neonates. Immature to 
TABLE 3: Audit of documentation perinatal risk factors.

\begin{tabular}{|c|c|c|c|}
\hline \multirow{2}{*}{$\begin{array}{l}\text { Perinatal risk factors present at birth } \\
\text { Frequency }(\mathrm{n}=320)(\%)\end{array}$} & \multicolumn{2}{|c|}{ Documented } & \multirow{2}{*}{ Not documented } \\
\hline & Present & Absent & \\
\hline Maternal fever $>38^{\circ} \mathrm{C}$ & $3(1)$ & $2(0.6)$ & $315(98.4)$ \\
\hline $\begin{array}{l}\text { Suspected or confirmed } \\
\text { Chorioamnionitis }\end{array}$ & $30(9.4)$ & $40(12.4)$ & $250(78.2)$ \\
\hline Prolonged rupture of membranes & $11(3.4)$ & $154(48.2)$ & $155(48.4)$ \\
\hline Difficult or prolonged labour & $9(2.8)$ & $16(5)$ & 295(92.2) \\
\hline Received intrapartum antibiotics & $2(0.6)$ & 0 & $318(99.4)$ \\
\hline Low birth weight $<2500 \mathrm{gms}$ & $155(48.4)$ & $165(51.6)$ & 0 \\
\hline
\end{tabular}

TABLE 4: Audit of investigations done on admission.

\begin{tabular}{lc}
\hline Investigations & $\begin{array}{c}\text { Frequency } \\
(\%)\end{array}$ \\
& $\mathrm{n}=320$ \\
\hline Complete blood count & $224(70)$ \\
C reactive protein & $198(62)$ \\
Immature to total neutrophil count & 0 \\
Blood culture & $13(4)$ \\
Lumbar puncture & 0 \\
Blood sugar & $26(8)$ \\
\hline
\end{tabular}

total neutrophil count and lumbar puncture were not done in any of the neonates.

3.5. Audit of Antibiotics Prescribing Practices. According to Kenyan guidelines (Basic Pediatrics Protocols - Feb 2016) recommended first-line treatment for neonatal sepsis is penicillin and gentamicin in combination. Appropriate antibiotics as per the Kenyan guidelines were prescribed in 313 (97.8\%) of neonates on admission. Ceftazidime and amikacin were prescribed in 7 neonates, while ceftazidime was prescribed for 1 neonate.

Appropriate doses of penicillin and gentamicin were given in 310 (96.9\%) and $282(88 \%)$, respectively, on admission. The dose of gentamicin for neonates weighing $<2 \mathrm{kgs}$ and aged $<7$ days is $3 \mathrm{mg} / \mathrm{kg}$. Overdose of gentamicin was observed in such babies.

According to Kenyan guidelines (Basic Paediatrics Protocols - Feb 2016), empiric antibiotics should be given for 48-72 hours and may be stopped if the neonate remained entirely well during this period. Table 5 shows antibiotic usage over 5 days.

At 48 hours, out of 276, 148 (53.62\%) neonates improved clinically, yet antibiotics were stopped only in $8(2.9 \%)$ and were changed to oral antibiotics in $6(2.17 \%)$. Thirty-four 34 (12.32\%) neonates deteriorated clinically and antibiotics were changed for 16 (5.8\%). Blood culture and CRP was done for $12(3.4 \%)$.

At 72 hours, out of 258, 168 (65.12\%) neonates improved clinically, yet antibiotics were stopped only in $22(8.53 \%)$ and were changed to oral antibiotics in $8(3.11 \%)$. Clinical deterioration was noted in 32 (12.4\%) neonates and antibiotics were

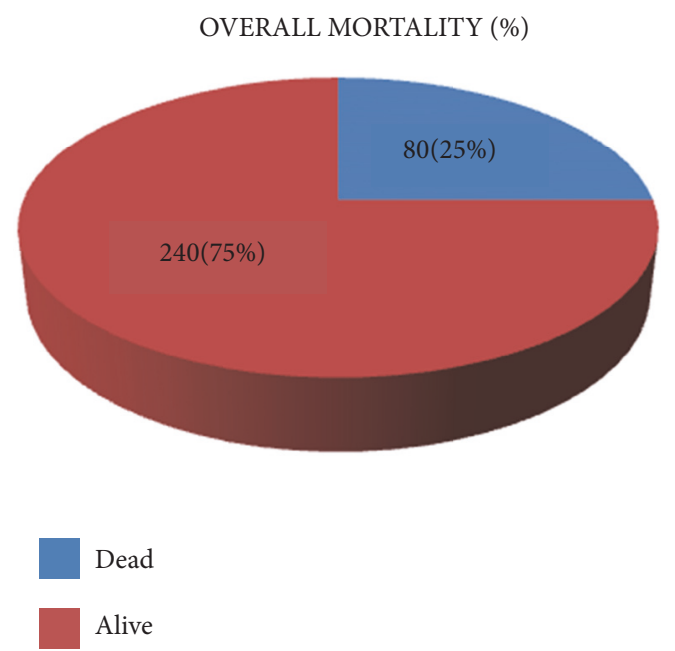

FIGURE 2: Overall mortality rate.

changed for 13 (5.03\%). Blood culture was done for 10 (3.9\%), while CRP was done for 16 (6.2\%).

The number of neonates decreases from 320 to 300 at 24 hours, to 276 at 48 hours, to 258 at 72 hours, and to 214 at 96 hours, and decreasing to 156 at 120 hours, either because the neonates died or discharged. The neonates were followed till discharge or death if it happened before 7 days.

3.6. Audit of Outcome at 7 Days. Overall mortality among 320 neonates admitted was 80 (25\%) over 7 days as shown in Figure 2. Mortality was high among preterm neonates; 70 (43.8\%) died out of 160 .

Figure 3 shows mortality rate according to time duration. Majority 44 (55\%) died within the first 48 hours and 36 (45\%) died between 48 hours and 7 days. Out of the 44 neonates who died within 48 hours, 38 (86.4\%) were preterm.

\section{Discussion}

This study was carried out to audit antibiotic prescribing practices for neonatal sepsis against recommended Kenyan guidelines in the New Born Unit of Kenyatta National Hospital. This was the first audit study done in this unit after the Kenyan guidelines were revised in Feb - 2016. 
TABLE 5: Audit of antibiotic usage over 5 days.

\begin{tabular}{|c|c|c|c|c|c|c|}
\hline & Frequency (\%) & $\begin{array}{c}\text { At } 24 \mathrm{hrs} \\
(\mathrm{n}=300)\end{array}$ & $\begin{array}{c}\text { At } 48 \text { hrs } \\
(\mathrm{n}=276)\end{array}$ & $\begin{array}{l}\text { At } 72 \text { hrs } \\
(\mathrm{n}=258)\end{array}$ & $\begin{array}{c}\text { At } 96 \text { hrs } \\
(\mathrm{n}=214)\end{array}$ & $\begin{array}{c}\text { At } 120 \text { hrs } \\
(\mathrm{n}=156)\end{array}$ \\
\hline \multirow{4}{*}{ Condition of the baby clinically } & Improved & $103(34.3)$ & $148(53.62)$ & $168(65.12)$ & $140(65.4)$ & $114(73.1)$ \\
\hline & Deteriorated & $29(9.7)$ & $34(12.32)$ & $32(12.4)$ & $24(11.21)$ & $12(7.7)$ \\
\hline & No change & $148(49.3)$ & $83(30.06)$ & $40(15.5)$ & $35(16.36)$ & $20(12.8)$ \\
\hline & Not documented & $20(6.7)$ & $11(4)$ & $18(6.98)$ & $15(7.01)$ & $10(6.4)$ \\
\hline \multirow{4}{*}{ Antibiotic usage } & Stopped & $1(0.35)$ & $8(2.9)$ & $22(8.53)$ & $34(15.9)$ & $40(25.65)$ \\
\hline & Continued & 298(99.3) & $246(89.13)$ & $215(83.33)$ & $156(72.9)$ & $106(67.95)$ \\
\hline & Changed & 0 & $16(5.8)$ & $13(5.03)$ & $16(7.5)$ & $5(3.2)$ \\
\hline & Changed to oral & $1(0.35)$ & $6(2.17)$ & $8(3.11)$ & $8(3.7)$ & $5(3.2)$ \\
\hline \multirow{2}{*}{ CRP done } & Yes & 0 & $12(3.4)$ & $16(6.2)$ & $10(4.7)$ & $6(3.8)$ \\
\hline & No & $300(100)$ & $264(96.6)$ & $242(93.8)$ & 204(95.3) & $150(96.2)$ \\
\hline \multirow{2}{*}{ Blood culture done } & Yes & 0 & $12(3.4)$ & $10(3.9)$ & $8(3.7)$ & $6(3.8)$ \\
\hline & No & $300(100)$ & $264(96.6)$ & $248(96.1)$ & 206(96.3) & $150(96.2)$ \\
\hline
\end{tabular}

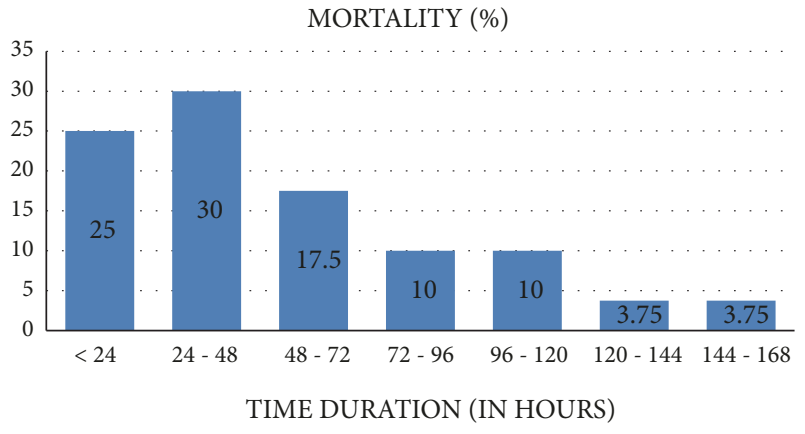

FIGURE 3: Mortality rate according to time duration.

Complete documentation of clinical features and perinatal risk factors for neonatal sepsis was not done in any of the 320 neonates. These features are clearly listed in the Basic Paediatric Protocols and should have been documented if there was continuous availability of a structured neonatal admission record. A structured neonatal admission form is in existence at $\mathrm{KNH}$ but availability had been erratic. It was presumed that some clinical features like convulsions, lethargy, and refusal to breastfeed were only documented if they were present. This appears to be a common Kenyan problem as reported by Kithinji et al. in the $\mathrm{KNH}$ general paediatric wards and [13] and Aluvaala et al, in 22 public hospitals of Kenya [14].

Diagnosis of neonatal infection largely depends on laboratory work-up. A full blood count and immature to total neutrophil ratio (I/T ratio) and C-reactive protein are some of the most available tests in $\mathrm{KNH}$. Unfortunately, I/T ratio is rarely done even when requested. Reagents for CRP are also sometimes out of stock. Our $4 \%$ rate of blood cultures was much lower than $14 \%$ reported by Simiyu et al. in an earlier study from the same NBU [15]. We did not audit availability of blood culture bottles during the current study but it could have contributed to the low levels reported. This is a recurrent problem in our institution. It is also possible that the attending clinician just did not consider doing this procedure. Ideally a neonate who is not responding to antibiotics should be reevaluated but the rate in our study was very low. The same reasons given above could be operational here. In Simiyu et al. study change of antibiotics was guided by culture and sensitivity reports in only $13.5 \%$ [15].

As for the blood sugar estimation the NBU is equipped with a glucometer and most of the times the strips for the test are available. This test therefore should have been done more frequently than reported in the current study if the admitting clinician ordered it.

In our study, appropriate antibiotics as per the Kenyan guidelines (Basic Pediatrics Protocols- 2016) were prescribed in $97.8 \%$ of neonates on admission. Appropriate doses of penicillin and gentamicin were given in $96.9 \%$ and $88 \%$, respectively, on admission. Overdose of gentamicin was observed in neonates weighing $<2 \mathrm{kgs}$ and aged $<7$ days. Our findings were better than Kithinji's study in $\mathrm{KNH}$ paediatric wards, where the recommended first line antibiotics were given in $64.4 \%$ of neonates, with $37.5 \%$ of them having error in the dosages. She also reported gentamicin overdosing in neonates $<7$ days and $<2 \mathrm{kgs}$ [13]. Aluvaala et al. also found error in dosages; about one in 10 of benzyl penicillin prescriptions was an overdose (11.6\%) in contrast to almost one in five (18.5\%) for gentamicin [14].

From this study we found that there was prolonged unnecessary use of antibiotics in neonates who improved clinically at $48-72$ hours just like in other studies done in high resource settings. Neonates who improved clinically at 48 hours were $53.62 \%$, yet antibiotics were stopped in $2.9 \%$ only. At 72 hours $65.12 \%$ neonates improved clinically, but antibiotics were stopped only in $8.53 \%$. The continuation of antibiotics was more inappropriately done than initiation of antibiotics. Earlier discontinuation of antibiotics was an issue, maybe because of inability to confirm infection. Other studies have reported similar results to ours.

Slogrove et al. in South Africa reported that 33.3\% of their infants were asymptomatic and treated empirically; the continuation of treatment beyond 24 hours was probably unnecessary in $38 \%$ of early and $28 \%$ of late antibiotic events without abnormal inflammatory markers at 48-72 hours. 
Neonates treated for $>6$ days during their first antibiotic event were more likely to require a second antibiotic event $(\mathrm{p}<0.02)$ [16]. Schellack et al. in South Africa also showed overuse of antibiotics; $58 \%$ of the antibiotics were administered for 10 days or longer [17]. An audit done by Abdelrhim et al., in United Kingdom in 2012, demonstrated all 22 could have been managed safely with $36 \mathrm{hrs}$ of antibiotics, but they were given antibiotics for $>48 \mathrm{hrs}[18]$. A Prospective Surveillance done by Cantey et al. in 2011-12 in Texas concluded that $94 \%$ of antibiotic use was empiric therapy for suspected infection. Only 63\% were discontinued after 48 hours, when cultures were sterile and $26 \%$ of antibiotics were continued for $\geq 5$ days despite sterile cultures; pneumonia $(16 \%)$ and culture-negative sepsis (8\%) were the major contributors [19]. A study done by Afjeh SA et al. in Iran also showed prolonged duration of antibiotic for 7-62 days with a mean duration of 24.01 days [20].

A retrospective cohort analysis of extremely low birth weight infants done by Cotton et al. concluded that the median duration of initial empirical antibiotic treatment varied significantly among centres, from 3 to 9.5 days $(\mathrm{P}<.001)$. In $27 \%-85 \%$ of neonatal centres empiric antibiotics had been administered for $>5$ days to neonates with negative cultures [21]. A multicentre study by Cordero and Ayers also reported that most neonates with suspected sepsis and negative blood cultures were given antibiotics, but no perinatal risk factors or clinical signs explained prolonged administration. Discontinuing empiric antibiotics when blood cultures are negative in asymptomatic extremely low birth weight infants can reduce antimicrobial exposure without compromising clinical outcome [22].

We should emphasize on discontinuing empiric antibiotics as soon as is feasible; if the neonate is clinically stable, blood cultures are negative and CRP is normal. There is a lack of well-designed clinical trials evaluating the appropriate duration of empirical antibiotics in blood-culture-negative sepsis. The only randomised controlled trial done by Saini et al. showed that there was no difference in the treatment failure (defined as reappearance of signs of sepsis within 15 days of stopping antibiotics, supported by laboratory evidence) rates between short course (48-96 hours) and long course (7 days) groups among neonates $>30$ weeks and $>1000$ grams with probable sepsis [23]. Randomised controlled trial by Gathwala et al. concluded that 10-day antibiotic therapy was as effective as 14-day therapy in blood culture-proven neonatal sepsis, if the neonate is clinically stable by day 7 of treatment [24].

In this study overall mortality was $80(25 \%)$ over 7 days. Of these 70 (87.5\%) were preterm neonates. Forty-four (55\%) neonates died within 48 hours. It is probable that most of these deaths were due to unavailability of respiratory support. Even though the NBU has facility for continuous positive airway pressure (CPAP) and mechanical ventilation, the numbers requiring this support outstrips availability. This is similar to what was reported by Musoke et al. in year 1992 even though at that time respiratory support was not available [25]. Simiyu et al. in 2004 in NBU at KNH reported a higher mortality rate of $57.4 \%$ [15]. Were et al. in KNH- NBU demonstrated neonatal survival rate of $62.6 \%$ in neonates born below 2000 grams. None of the neonates born less than 1000 grams survived the neonatal period. They reported that over $28 \%$ of the mortality occurring within the first 24 hours, which is comparable to our study, could be due to lack of intensive care facilities and inadequate obstetric services [26].

A prospective study done in Egypt showed mortality rate of $51 \%$ for proven early-onset sepsis and $42.9 \%$ for proven late-onset sepsis [27]. A prospective study by Baqui et al. in India also reported high mortality in prematurity. In their study, $74.8 \%$ of deaths occurred due to prematurity in the first week of life, with $30 \%$ in the first 24 hours, and $>50 \%$ of neonatal deaths secondary to sepsis occurred in the first week of life [28].

\section{Study Limitation}

This study being an audit of medical records only reflected what was documented. Also unavailability of the structured neonatal admission record for all admissions as clinicians only reported the positive responses. Hawthorne effect: people might have changed their practices when they came to know about the study.

\section{Conclusion}

There was poor documentation of clinical features, perinatal risk factors, and condition of the neonates at the time of change of antibiotics. Appropriate antibiotics as per the Kenyan guidelines were given in $97.8 \%$ of neonates on admission. The rate of investigations to confirm infection was very low. Blood cultures were done only in $4 \%$ of neonates on admission and lumbar punctures were not done. The continuation of antibiotics was inappropriate. Overall mortality was high in neonates at $80(25 \%)$. Most neonates died within 48 hours and majority were preterm.

\section{Additional Points}

Recommendations. This study sets a baseline for continued audit and change of practice in the NBU. To improve documentation, a structured neonatal admission record should be available at all times. Adequate follow-up of all babies on antibiotic as per the Kenyan guidelines is needed.

\section{Data Availability}

The data used to support the findings of this study are available from the corresponding author upon request.

\section{Conflicts of Interest}

The authors declare no conflicts of interest.

\section{Acknowledgments}

We would like to thank the research assistants for helping in collecting data and Professor D Wamalwa for guidance in 
this study and Dr. Hussein Dosajee for analysing the data. We thank KNH for allowing us to carry out this study.

\section{References}

[1] WHO, "Newborns: reducing mortality," http://www.who.int/ mediacentre/factsheets/fs333/en/.

[2] A. C. Seale, H. Blencowe, A. Zaidi et al., "Neonatal severe bacterial infection impairment estimates in South Asia, subSaharan Africa, and Latin America for 2010," Pediatric Research, vol. 74, no. 1, pp. 73-85, 2013.

[3] M. Kohli-Lynch, N. J. Russell, A. C. Seale et al., "Neurodevelopmental impairment in children after group B streptococcal disease worldwide: systematic review and meta-analyses," Clinical Infectious Diseases, vol. 65, pp. S190-S199, 2017.

[4] B. E. Stephens and B. R. Vohr, "Neurodevelopmental outcome of the premature infant," Pediatric Clinics of North America, vol. 56, no. 3, pp. 631-646, 2009.

[5] K. M. Rand, N. C. Austin, T. E. Inder, S. Bora, and L. J. Woodward, "Neonatal infection and later neurodevelopmental risk in the very preterm infant," Journal of Pediatrics, vol. 170, pp. 97-104, 2016.

[6] G. Klinger, I. Levy, L. Sirota, V. Boyko, L. Lerner-Geva, and B. Reichman, "Outcome of early-onset sepsis in a national cohort of very low birth weight infants," Pediatrics, vol. 125, no. 4, pp. e736-e740, 2010.

[7] N. Tripathi, C. M. Cotten, and P. B. Smith, "Antibiotic use and misuse in the neonatal intensive care unit," Clinics in Perinatology, vol. 39, no. 1, pp. 61-68, 2012.

[8] R. N. Musoke and G. Revathi, "Emergence of multidrugresistant Gram-negative organisms in a neonatal unit and the therapeutic implications," Journal of Tropical Pediatrics, vol. 46, no. 2, pp. 86-91, 2000.

[9] A. B. Russell, M. Sharland, and P. T. Heath, "Improving antibiotic prescribing in neonatal units: Time to act," $A D C$ Fetal and Neonatal Edition, vol. 97, no. 2, pp. F141-F146, 2012.

[10] V. S. Kuppala, J. Meinzen-Derr, A. L. Morrow, and K. R. Schibler, "Prolonged initial empirical antibiotic treatment is associated with adverse outcomes in premature infants," Journal of Pediatrics, vol. 159, no. 5, pp. 720-725, 2011.

[11] “Basic Paediatric Protocol 2016," http://www.idoc-africa.org/ index.php/102-home/144-bpp.

[12] H. N. Nagesh, P. L. Basavanna, and M. R. Savitha, "Antibiotic usage and auditing of antibiotic sensitivity pattern of culture positive neonatal septicemia in neonatal intensive care unit of a tertiary care hospital: a retrospective study," International Journal of Contemporary Pediatrics, vol. 1, no. 3, pp. 142-147, 2017.

[13] B. N. Kithinji, Audit of Quality of Neonatal Sepsis Care at Kenyatta National Hospital General Paediatric Wards, University of Nairobi, 2014, http://erepository.uonbi.ac.ke:8080/xmlui/ handle/11295/76154.

[14] J. Aluvaala, R. Nyamai, F. Were et al., "Assessment of neonatal care in clinical training facilities in Kenya," Archives of Disease in Childhood, vol. 100, no. 1, pp. 42-47, 2015.

[15] D. E. Simiyu, "Morbidity and mortality of low birth weight infants in the new born unit of Kenyatta National Hospital, Nairobi," East African Medical Journal, vol. 81, no. 7, pp. 367$374,2004$.

[16] A. L. Slogrove, S. Holgate, M. F. Cotton, H. Rabie, H. M. Kunneke, and A. Engelbrecht, "Evaluating antibiotic use in a secondary level hospital neonatal unit in the Western Cape, South Africa," Southern African Journal of Epidemiology and Infection, vol. 25, no. 3, pp. 21-25, 2010.

[17] N. Schellack and A. G. Gous, "Antibiotic prescribing patterns in a neonatal intensive care unit," Southern African Journal of Epidemiology and Infection, vol. 26, no. 4, pp. 267-270, 2011.

[18] H. Abdelrhim, P. A. Heaton, C. Routley, and S. P. Paul, "Treatment for early-onset neonatal sepsis," Nursing Times, vol. 110, no. 14, pp. 24-25, 2014.

[19] J. B. Cantey, P. S. Wozniak, and P. J. Sánchez, "Prospective surveillance of antibiotic use in the neonatal intensive care unit," The Pediatric Infectious Disease Journal, vol. 34, no. 3, pp. 267272, 2015.

[20] S. Afjeh, M. Sabzehei, S. Fahimzad, F. Shiva, A. Shamshiri, and F. Esmaili, "Antibiotic Therapy for Very Low Birth Weight Newborns in NICU," Iranian Journal of Pediatrics, vol. 26, no. 2, 2016.

[21] C. Michael Cotten, S. Taylor, B. Stoll et al., "Prolonged duration of initial empirical antibiotic treatment is associated with increased rates of necrotizing enterocolitis and death for extremely low birth weight infants," Pediatrics, vol. 123, no. 1, pp. 58-66, 2009.

[22] L. Cordero and L. W. Ayers, "Duration of empiric antibiotics for suspected early-onset sepsis in extremely low birth weight infants," Infection Control and Hospital Epidemiology, vol. 24, no. 9, pp. 662-666, 2003.

[23] S. S. Saini, S. Dutta, P. Ray, and A. Narang, "Short course versus 7-day course of intravenous antibiotics for probable neonatal septicemia: A pilot, open-label, randomized controlled trial," Indian Pediatrics, vol. 48, no. 1, pp. 19-24, 2011.

[24] G. Gathwala, A. Sindwani, J. Singh, O. Choudhry, and U. Chaudhary, "Ten days vs. 14 days antibiotic therapy in cultureproven neonatal sepsis," Journal of Tropical Pediatrics, vol. 56, no. 6, pp. 433-435, 2010.

[25] E. Kasirye-Bainda and F. N. Musoke, "Neonatal morbidity and mortality at Kenyatta National Hospital newborn unit." East African Medical Journal, vol. 69, no. 7, pp. 360-365, 1992.

[26] F. N. Were, B. O. Mukhwana, and R. N. Musoke, "Neonatal survival of infants less than 2000 grams born at Kenyatta National Hospital," East African Medical Journal, vol. 79, no. 2, pp. 77-79, 2002.

[27] E. M. R. Shehab El-Din, M. M. A. El-Sokkary, M. R. Bassiouny, and R. Hassan, "Epidemiology of neonatal sepsis and implicated pathogens: a study from Egypt," BioMed Research International, vol. 2015, Article ID 509484, 11 pages, 2015.

[28] A. H. Baqui, G. L. Darmstadt, E. K. Williams et al., "Rates, timing and causes of neonatal deaths in rural India: Implications for neonatal health programmes," Bulletin of the World Health Organization, vol. 84, no. 9, pp. 706-713, 2006. 


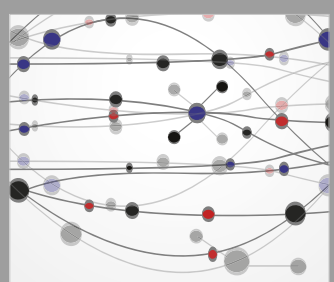

The Scientific World Journal
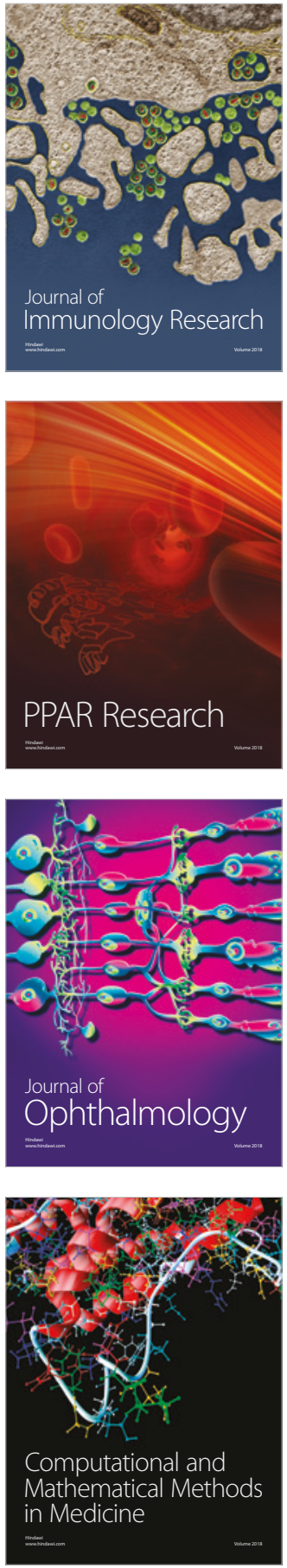

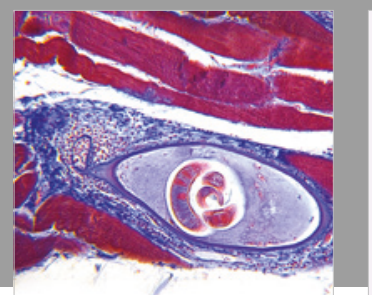

Gastroenterology Research and Practice

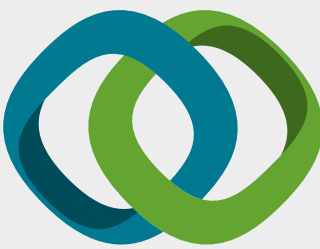

\section{Hindawi}

Submit your manuscripts at

www.hindawi.com
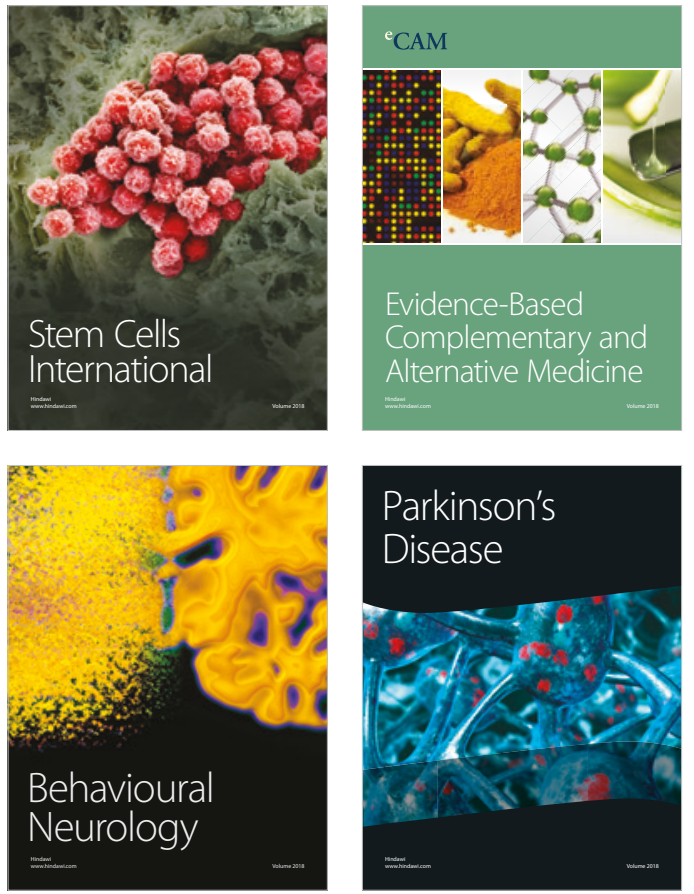

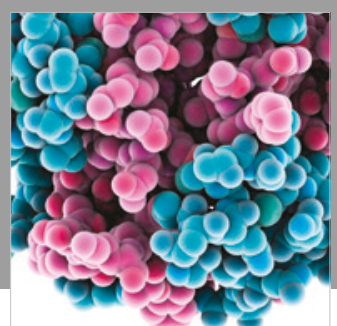

ournal of

Diabetes Research

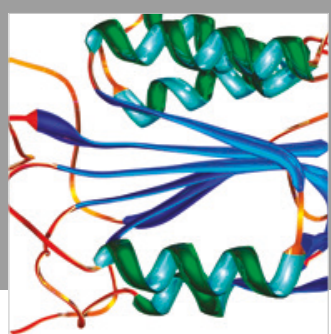

Disease Markers
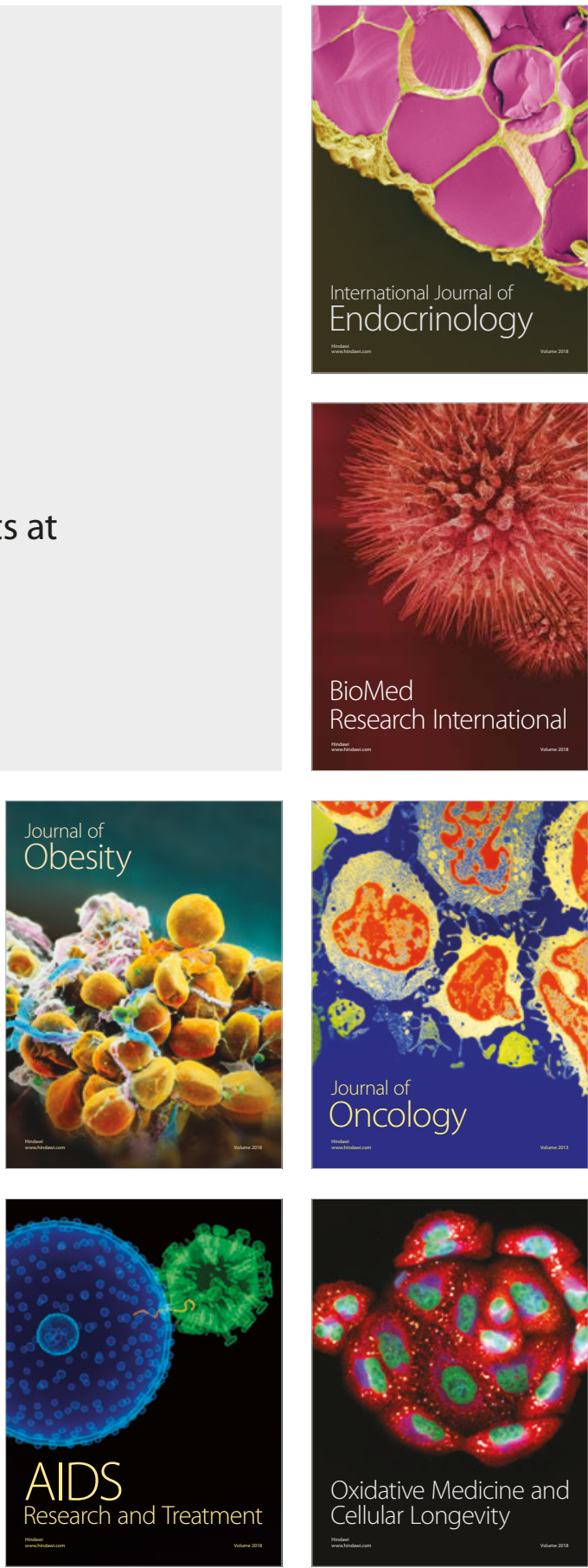